\title{
Conceptual and physical differences in the category effect
}

\author{
PETER DIXON and JUDITH M. SHEDDEN \\ University of Alberta, Edmonton, Alberta, Canada
}

\begin{abstract}
In these experiments we tested whether physical differences between letters and digits could account for the category effect in visual detection and partial report. In our task, observers decided whether a target item matched any item in a briefly presented array. This yielded a visual detection task when the target preceded the array and a type of partial-report task when the target followed the array. In Experiment 1, the stimulus set consisted of nine digits and nine letters modified to match the digits on the basis of visual similarity. Partial-report performance was better in a mixed-category condition than in a single-category condition, but no such effect occurred in visual detection. However, the similarity of items between categories may have biased observers against using category information to perform the task. When the similarity was controlled both within and between categories in Experiment 2, a category effect emerged in visual detection as well. A third experiment, using a same-different reaction time task, verified that the stimuli were equally similar within and between categories. The results indicate that physical differences alone cannot explain the category effect.
\end{abstract}

It has been found in a number of tasks that the visual processing of an alphanumeric display is more efficient when some of the items come from an irrelevant category. For instance, it takes less time to find a digit in an array of letters than in an array of other digits (e.g., Egeth, Jonides, \& Wall, 1972), and detection of a digit in a brief display is more accurate when the distractors are letters rather than digits (e.g., Schneider \& Shiffrin, 1977). This finding can be referred to as a category effect. The category effect is important in theories of visual information processing because it has suggested to some that, at some level, characters are processed in parallel with unlimited capacity (e.g., Duncan, 1980; Gardner, 1973). However, this conclusion has not been universally accepted, and the mechanism underlying the category effect remains controversial (Deutsch, 1977; Kahneman \& Treisman, 1984; Krueger, 1984). In this study, we tested an often proposed counterexplanation of the category effect that can be termed the partial analysis account.

The unlimited parallel processing view of the category effect assumes that the items in a visual display are identified and categorized in parallel without requiring effort or attention on the part of the observer. However, such subsequent operations as transferring items to short-term memory or comparing items may limit performance. In this view, the category effect occurs because information about the category of items in the visual display can be used to separate relevant from irrelevant items. For instance, if the observers in a visual detection task know

This research was supported by Natural Sciences and Engineering Research Council of Canada Grant A8263 to the first author. We would like to thank Vince Di Lollo for comments on an earlier draft. Requests for reprints should be sent to Peter Dixon, Department of Psychology, University of Alberta, Edmonton, AB T6G 2E9, Canada. that the target is a digit, there is no need to perform any further operations on items that have already been classified as letters. Performance improves with mixed-category displays, then, because the observer can attend to relevant category items, and avoid spending processing resources on irrelevant items.

The partial analysis interpretation is that it is possible to distinguish the relevant items from the irrelevant items on the basis of a few visual features without performing any abstract categorization. For instance, in a given experiment, digits may tend to be more rounded than letters. If this is the case, observers should be able to improve their performance with mixed-category displays by paying special attention to items with curves. Although such a strategy may not serve to distinguish all letters from all digits, it may be sufficient to improve performance relative to a condition with only digits. The partial analysis explanation holds that, on average, item category can be ascertained on the basis of a partial analysis of the item's visual features. This account seems capable of explaining the category effect without hypothesizing an unlimited capacity for categorizing characters.

Several researchers have attempted to discount the partial analysis account by demonstrating a category effect after controlling for the feature differences between letters and digits. For example, Duncan (1983b) used a partial-report task with two kinds of character sets. In the first set, digits and letters were closely matched in terms of such features as curves, closure, and vertical lines, and in the second set, a single diagnostic feature allowed the two categories to be distinguished. Duncan found a similar category effect with both character sets, suggesting that the presence of diagnostic features was not important. Ingling (1972) also found a category effect in visual search after matching letters to digits on the "overall 
physical characteristics" (Ingling, 1972, p. 241). Jonides and Gleitman (1972) found a category effect with the digit zero as a target and letters as the distractors, but no category effect when the target was described as the letter "oh," even though identical visual displays were used (but see Duncan, 1983a). If there were some features that distinguished zero from letters, one might expect those features to be used to distinguish "oh" from other letters as well.

In many of these demonstrations it is possible that the control for featural differences was not complete. As long as the nature of visual feature analysis is unknown, one can argue that there was some other set of features that was used to distinguish letters and digits. Such features could be quite subtle, such as the presence of certain types of serifs, or relatively abstract and complex, such as the relationship between curves and straight lines. Moreover, the features used to distinguish the categories need not be perfectly reliable; a moderate correlation between a few features and item category may be sufficient to produce the effect.

A recent article by Krueger (1984) suggests that this skepticism is warranted, and that the partial analysis account may be correct. He used a visual search task in which subjects searched for a single item in a circular array of 2,4 , or 6 characters. The similarity of letters and digits was controlled by matching each digit with a visually similar letter and using only those items as stimuli. For example, 5 was matched with $S, 6$ with $G$, and so on. Krueger found no hint of a category effect in reaction time when visual similarity was controlled in this way. He concluded that the category effect found in other studies depended on the visual feature differences between the two categories.

Because the category effect has played a key role in theories of visual information processing, it is important to determine whether Krueger's (1984) finding also holds in paradigms other than visual search. For instance, previous research has found category effects in visual detection, when subjects try to detect a target in a brief display (Duncan, 1980; Schneider \& Shiffrin, 1977), and in the partial-report task, in which subjects are shown a brief display of items and are then probed to report some portion of it (Duncan, 1983b; Merikle, 1980). In the present study, we used a task developed by Di Lollo and Moscovitch (1983) that combines elements of both visual detection and partial report.

In this task, observers were first shown briefly a single target item and an array of items and then had to decide whether the target item was also present in the array. The target could either precede or follow the array. When the target preceded the array, the task was essentially a visual detection task; when the target followed the array, the task resembled a partial-report task in which observers were cued on the basis of item identity (Townsend, 1973). Previous studies had found large and robust category effects in this task, both when the target followed the array and when it preceded the array (Di Lollo \& Moscovitch, 1983;
Dixon, 1985). The present study investigated whether this category effect still would occur when stringent controls were placed on visual feature differences between categories. A positive result would suggest that the partial analysis account of the category effect is inadequate, and would support alternatives such as unlimited parallel processing.

\section{EXPERIMENT 1}

In the first experiment, the visual feature control used by Krueger (1984) was applied to the target-array task of Di Lollo and Moscovitch (1983) and Dixon (1985). If there was no difference between single-category displays and mixed-category displays, it would be strong evidence that the category effect was due to feature differences between letters and digits. Previous work with the category effect demonstrated that the effect is most likely to occur when there is only a single relevant item in the display (Dixon, 1985; Duncan, 1980, 1983b). Consequently, in the mixed-category displays in this experiment there were six irrelevant category items (letters) and one relevant category item (a digit). A category effect would be demonstrated if performance was better with these mixedcategory displays than with single-category displays of seven digits.

Matching each digit with a visually similar letter may not be sufficient to eliminate feature differences between categories. Even though there are only small differences between the letter and digit in a given pair, those differences may be consistent across pairs, and the categories could still be distinguished on the basis of those differences. Consequently, the characters were modified so that the letter-digit differences varied across pairs. The character set is shown in Figure 1. With these stimuli it seemed unlikely that category could be distinguished effectively on the basis of a few visual features.

It should be emphasized that this manipulation provides a conservative test of whether abstract category information is used in this task. The characters within a pair are very similar and difficult to tell apart with a brief exposure. Thus, there is much more visual similarity between categories than there is within categories. If observers have difficulty in distinguishing the category of a given item, it is likely to make a strategy of attending to category information unattractive (cf. Corcoran \& Jackson, 1977). Thus, if a category effect occurs, it would be strong evidence against any partial analysis explanation.

\section{Method}

Each trial contained two brief displays separated by a variable stimulus-onset asynchrony (SOA). One display was the stimulus array, which consisted of seven alphanumeric characters arranged in a circle. The other display was the target item, which was presented in the center of the circle. The observer's task was to decide whether the target character matched one of the characters in the array, and to press either a present or an absent response switch. The SOA between the array and the target was $-500,0$, 

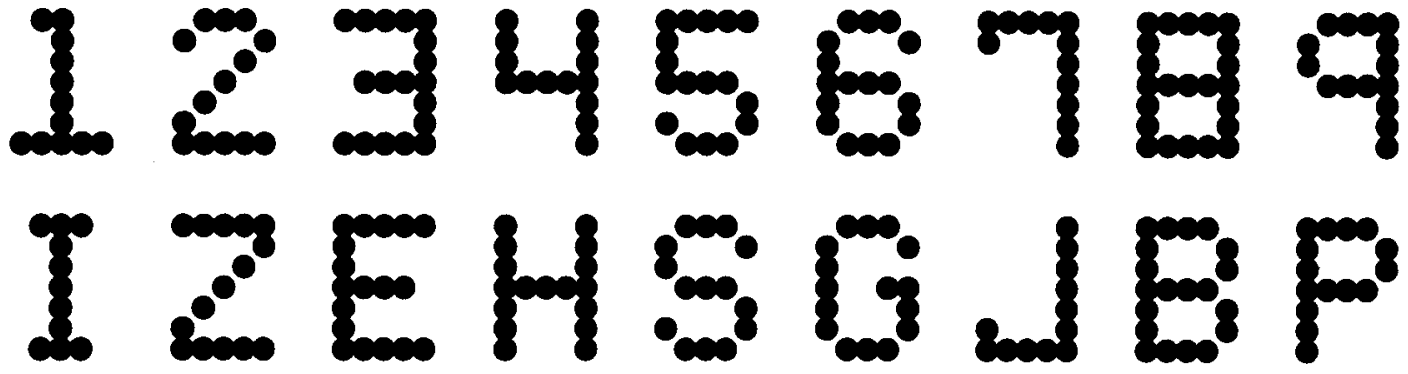

Figure 1. Stimulus set used in Experiment 1. The letter matched to each digit is shown below it.

or $500 \mathrm{msec}$. By convention, a negative SOA indicates a trial in which the target precedes the array and a positive SOA indicates a trial in which the target follows the array. At 0-msec SOA, the two displays are shown simultaneously.

The stimuli were displayed on a 30 -cm black-and-white video monitor at a distance of about $70 \mathrm{~cm}$. At that distance, characters subtended about $0.3^{\circ}$ of visual angle horizontally and about $0.4^{\circ}$ vertically. The experiment was run in a semi-illuminated room with a space-average luminance of about $9 \mathrm{~cd} / \mathrm{m}^{2}$. The monitor was adjusted so that the characters were displayed at near maximum contrast $[100 \times(\mathrm{L} \max -\mathrm{L} \min ) /(\mathrm{L} \max +\mathrm{Lmin})=95 \%$ contrast $]$. The space-average luminance of the white background field on the monitor was about $229 \mathrm{~cd} / \mathrm{m}^{2}$. Both target and array were shown for a single video raster scan (about $17 \mathrm{msec}$ ).

The procedure on each trial was as follows: When the computer was ready, a rectangular white fixation field $2.8^{\circ} \times 3.3^{\circ}$ was displayed. The target and array were shown, black on white, centered in this field. The trial began when the observer pressed both of the response switches in a hand-held response box. After $500 \mathrm{msec}$, the target and the array were presented with the appropriate SOA. The fixation field remained on the screen until the observer responded by pressing either the present response switch or the $a b$ sent response switch, after which the screen was blank. There was a pause of about $1 \mathrm{sec}$ between trials. At the end of each block of trials, the observer received feedback about the overall accuracy in that block.

There were four types of trial blocks resulting from the factorial combination of two factors. The first factor was array composition. In single-category blocks, the array consisted of seven different randomly selected digits. In mixed-category blocks, the array consisted of one digit and six letters. The target was a digit in both kinds of blocks. Mixed-category displays were constructed by substituting the matched letter for six of the distractor digits in a singlecategory array (see Figure 1). This ensured that, on mixed-category trials, the letter matched to the target digit was not used as a distractor in the array. The second factor was the radius of the circular array. In half of the blocks, the array items were $0.6^{\circ}$ from the center of the display (with adjacent items being $0.3^{\circ}$ apart); in the other half, they were $0.9^{\circ}$ from the center (with adjacent items being $0.4^{\circ}$ apart). This factor will not be discussed further because it had no overall effect and did not interact with any other factors.

Each block consisted of 48 randomly ordered trials in which each of the three SOAs was used an equal number of times. Half the trials were present trials, in which the target matched a digit in the array, and half were absent trials, in which there was no match for the target. Observers were informed about the nature of each block before it began, and were given 10 randomly selected practice trials before each block. The observers were first given one each of the four kinds of blocks in one order, and then given a second set of the four kinds of blocks in reversed order. Across observers, each type of block occurred in each serial position in the experimental session an equal number of times. The entire session lasted about $50 \mathrm{~min}$. Three observers were run with each of four different block orders, making a total of 12 observers, all undergraduates at the University of Alberta.

The primary analysis was conducted on a nonparametric measure of sensitivity, $A^{\prime}$, calculated for each observer, condition, and SOA (Grier, 1971; Pollack \& Norman, 1964). A' can be interpreted as the area under the receiver operating characteristic, and is equivalent to the proportion correct that would result if a two-alternative forced-choice paradigm were used (Green \& Swets, 1966). Parallel analyses were conducted on percent correct on present and absent trials as well. Although responses were not speeded, analyses were also conducted on median correct response time in each condition.

\section{Results}

As shown in Figure 2, there was an overall effect on $A^{\prime}$ of single versus mixed category $[F(1,11)=20.87$, $p<.001]$. This effect, however, occurred only at the +500 -msec SOA $[F(1,8)=32.40, p<.001]$, and was not significant at -500 or 0 msec. This was reflected in

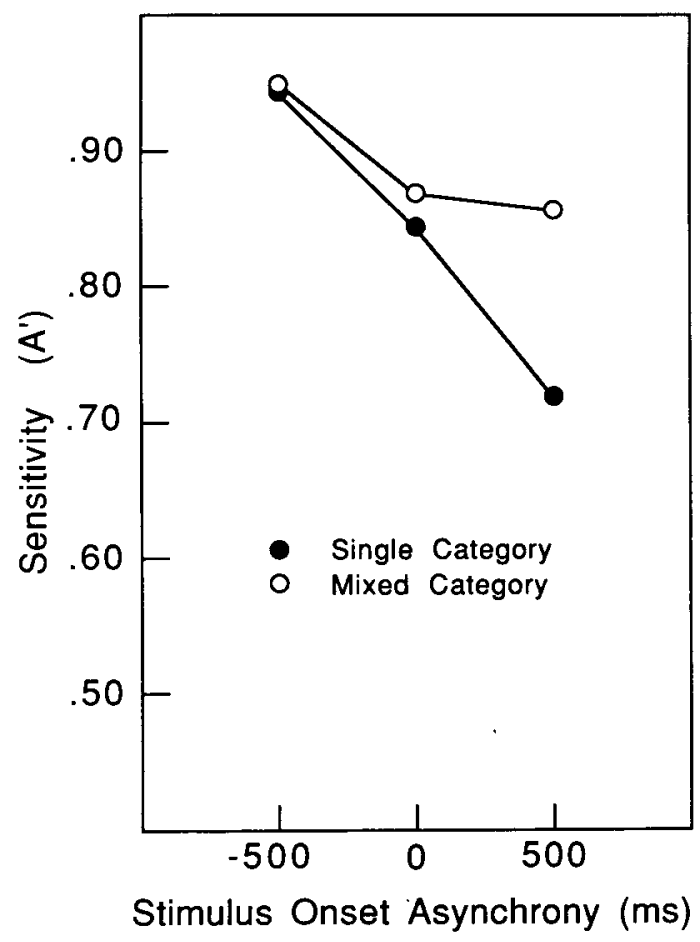

Figure 2. Mean $A^{\prime}$ results from Experiment 1. 
a significant interaction between category condition and SOA $[F(2,16)=13.21, p<.001]$. There was also an overall effect of SOA $[F(2,22)=52.96, p<.001]$, with accuracy declining as SOA increased. Previous research using this task has found a nonmonotonic effect of SOA, with accuracy being worst at SOAs of 100-200 msec and improving with longer SOAs (Di Lollo \& Moscovitch, 1983; Dixon, 1985, 1986). It is likely that such a trend would have been apparent in the present experiment as well if SOAs of 100 and $200 \mathrm{msec}$ had been included.

Percent correct for present and absent trials (i.e., the hit and correct-rejection rates) are shown in Table 1 . As with the $A^{\prime}$ scores, there was an effect of category condition $[F(1,11)=16.17, p<.005]$, an effect of SOA $[F(2,22)=67.40, p<.001]$, and an interaction between the two $[F(2,22)=8.65, p<.005]$. There was no effect or interaction involving present/absent trials.

Response times are also shown in Table 1 . There was an overall effect of SOA $[F(2,22)=17.06, p<.001]$, with response time being longest at 0 -msec SOA. Absent responses took longer than present responses $[F(1,11)=$ $72.61, p<.001]$, particularly at 0 -msec SOA $[F(2,22)$ $=3.56, p<.05]$. There was no overall difference between single- and mixed-category trials $[F(1,11)<1]$, but single-category trials tended to be slower at $500-\mathrm{msec}$ SOA, leading to a significant interaction between SOA and single versus mixed category $[F(2,22)=5.37$, $p<.05]$.

\section{Discussion}

This experiment demonstrated a substantial category effect when the target followed the array, but little or none when the target preceded or coincided with the array. The category effect has different characteristics in these two situations. When the array is presented first, knowing the

Table 1

Response Times (in Milliseconds) and Percent Accuracy in Experiment 1

\begin{tabular}{|c|c|c|c|}
\hline $\begin{array}{c}\text { Stimulus } \\
\text { Onset } \\
\text { Asynchrony } \\
\end{array}$ & Trial Type & Response Time & Accuracy \\
\hline \multicolumn{4}{|c|}{ Single Category } \\
\hline$-500 \mathrm{msec}$ & $\begin{array}{l}\text { Present } \\
\text { Absent }\end{array}$ & $\begin{array}{l}707 \\
927\end{array}$ & $\begin{array}{l}89.6 \\
93.0\end{array}$ \\
\hline $0 \mathrm{msec}$ & $\begin{array}{l}\text { Present } \\
\text { Absent }\end{array}$ & $\begin{array}{r}854 \\
1182\end{array}$ & $\begin{array}{l}78.4 \\
74.0\end{array}$ \\
\hline $500 \mathrm{msec}$ & $\begin{array}{l}\text { Present } \\
\text { Absent }\end{array}$ & $\begin{array}{r}778 \\
1056\end{array}$ & $\begin{array}{l}68.8 \\
58.9\end{array}$ \\
\hline \multicolumn{4}{|c|}{ Mixed Category } \\
\hline$-500 \mathrm{msec}$ & $\begin{array}{l}\text { Present } \\
\text { Absent }\end{array}$ & $\begin{array}{l}703 \\
983\end{array}$ & $\begin{array}{l}89.6 \\
91.4\end{array}$ \\
\hline $0 \mathrm{msec}$ & $\begin{array}{l}\text { Present } \\
\text { Absent }\end{array}$ & $\begin{array}{r}831 \\
1242\end{array}$ & $\begin{array}{l}76.4 \\
81.0\end{array}$ \\
\hline $500 \mathrm{msec}$ & $\begin{array}{l}\text { Present } \\
\text { Absent }\end{array}$ & $\begin{array}{l}679 \\
961 \\
\end{array}$ & $\begin{array}{l}80.5 \\
75.3\end{array}$ \\
\hline
\end{tabular}

relevant category aids in deciding which array items should be retained in memory until the target is presented. Thus, the category effect in the present data suggests that retaining one item from the array is less demanding than retaining seven. When the target is presented first, the category effect has a somewhat different interpretation. Knowing the relevant category in this case should help decide which items should be compared with the target. Presumably, the comparison process would be more efficient if most items could be dismissed as irrelevant prior to comparison with the target. The failure to find a category effect in the present data suggests that this improvement in efficiency may not offset whatever cost there is in the use of category information.

The present results are consistent with those of Duncan (1983a). He found no difference in response time between searching for the letter "oh" among digits and searching for the digit "zero" among digits, thus failing to replicate earlier work by Jonides and Gleitman (1972). Duncan concluded that an effect of category, independent of physical differences, could be obtained in visual search only when there was more than one item in the set of potential targets. Presumably, having more potential targets to search for would make the comparison process more demanding and the strategy of using category information to eliminate irrelevant items more attractive. In sum, the results are broadly consistent with the conclusion that a category effect occurs with partial report (for which all of the relevant items must be retained, regardless of the identity of the target) but not with visual detection (for which a single given item must be found).

However, there are at least two reasons to be skeptical of this conclusion. One problem is that items in the mixedcategory arrays may have been more difficult to identify than those in single-category arrays. For each letter in a mixed-category array, there was at least one potential item that was likely to be confused with it, namely, the digit to which it was visually matched. This was not the case with single-category arrays. Because observers knew that all of the array items were digits, they would be unlikely to interpret any of them as letters. Essentially, the items were more confusable between categories than they were within categories. Because at least one likely visual confusion that could occur in the mixed-category condition could not occur in the single-category condition, the mixed-category condition may have been more demanding.

A second problem is that a category effect may have been masked by a ceiling effect. That is, the conditions that failed to show a category effect are precisely those that had the highest overall accuracy. In these cases, the task may have been so easy that there was little incentive to use category information. Moreover, any small effects of category information that did occur would be difficult to detect statistically. For these reasons, it seems likely that Experiment 1 was not a fair test of the category effect at $-500-m s e c$ SOA. This possibility was pursued in Experiment 2. 


\section{EXPERIMENT 2}

Experiment 2 further investigated the category effect in visual detection (i.e., on negative SOA trials) with stimuli that were equally similar within and between catgories. As pointed out previously, Experiment 1 may have been a conservative test of the effect because items may have been more similar between categories than within categories. In the present experiment, the set of stimulus items was constructed by putting items together in groups of two digits and two letters. Each digit in a given group was matched with a visually similar letter, as in Experiment 1 , but, in addition, it was matched to the other digit in the group. Thus, for each digit there was a visually confusable letter and a visually confusable digit. Four groups of items were constructed as shown in Figure 3. The intention was that each digit-letter pair (the columns in each group in Figure 3) as well as each digit-digit pair (the upper row in each group) would differ by only one or two features. If a visual detection category effect occurred with this stimulus set, it would suggest that the manipulation in Experiment 1 was too conservative, and was not a fair test of the effect.

In addition, two other changes were made to correct possible problems that might have occurred in Experiment 1 . First, the number of items in the array was increased from seven to nine to lessen the influence of ceiling effects. Second, the number of items confusable with the target was equated across the mixed- and singlecategory conditions. In both conditions, only one item in the array was from the same group of four as the target
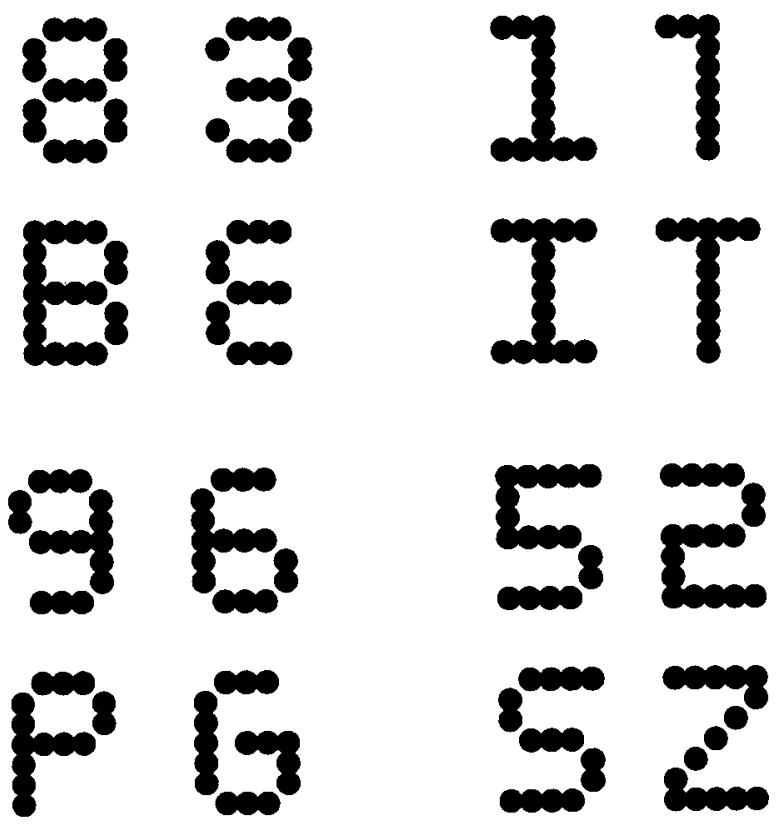

Figure 3. Stimulus set used in Experiment 2. Matched digits are shown next to each other, and the letters matched to the digits are shown below them. item. On present trials, this was the target itself, and on absent trials, this was the digit matched to the target. All other array items came from the other three groups and presumably had a relatively low likelihood of being confused with the target.

\section{Method}

Stimuli in Experiment 2 were constructed from the items shown in Figure 3. Because the form of these letters and digits was somewhat novel, observers first participated in a familiarization task. This consisted of a same-different task in which a single item was shown on the screen while the name of an item was presented auditorily. The procedure on each trial of the familiarization task was as follows. The trial began with the presentation of a white fixation field $2.0^{\circ}$ wide and $1.3^{\circ}$ high. Observers initiated presentation of the stimuli by pressing both response switches in a handheld response box. After $500 \mathrm{msec}$, an item was presented centered in the fixation field. At the same time, the name of an item was presented over a loudspeaker at a comfortable listening volume. The visual display remained in view until the observer responded by pressing either the same response switch on the right or the different response switch on the left. Auditory feedback was presented in the case of an error. The display and viewing conditions were the same as in Experiment 1. The names were generated by a Jameco Model JE520 voice synthesizer. Each observer completed two blocks of trials consisting of 32 same trials and 48 different trials in a random order. On different trials, the visual and auditory items always came from the same group of four items.

After the familiarization task, the observers performed the detection experiment. The stimuli in this task were constructed as follows. First, one of the four groups of items was designated as the target group and one of the two digits in the group was selected as the target digit. The arrays contained nine items, only one of which was from the target group. On present trials, this was the target digit, and on absent trials, this was the digit matched to the target. In the single-category condition, the other eight items in the array were a random selection of digits from the other three groups, subject to the constraints that each of the six digits appear at least once in the array and that adjacent items not be identical. In the mixed-category condition, the other eight items were selected similarly, except that the letters from each group were used instead of the digits. Thus, the only difference between single- and mixedcategory arrays was whether items from the nontarget groups were letters or digits.

In both conditions, the array items were arranged in a $1.2^{\circ}$-diam circle and the SOA was $-600 \mathrm{msec}$, with the target preceding the array. Otherwise, the procedure and apparatus were the same as in Experiment 1. The observers received two practice blocks followed by six test blocks of 32 trials each, in which each digit appeared as a target an equal number of times. Blocks alternated between the single category and the mixed-category conditions, with half of the observers beginning with the single-category condition and half beginning with the mixed-category condition. Each block began with 10 practice trials. Ten undergraduates from the University of Alberta served as the observers.

\section{Results and Discussion}

There was a robust advantage of the mixed-category over the single-category condition $\left[A^{\prime}=.909\right.$ vs. $A^{\prime}=.847$; $F(1,9)=21.64, p<.005]$, with all observers showing the effect. This was significantly larger than the effect obtained at $-500 \mathrm{msec}$ in Experiment $1[t(20)=3.52$, $p<.005]$. Thus, the category effect seems to occur in visual detection even when the two categories cannot be 
Table 2

Response Times (in Milliseconds) and Percent Accuracy in Experiment 2

\begin{tabular}{ccc}
\hline Trial Type & Response Time & Accuracy \\
\hline & Single Category & \\
Present & 666 & 84.4 \\
Absent & 933 & 66.5 \\
& & \\
Present & Mixed Category & \\
Absent & 678 & 97.7 \\
\hline
\end{tabular}

distinguished on the basis of physical features. The effect seems to require only that items be equally confusable within and between categories. The fact that no category effect was found in Experiment 1 at -500 -msec SOA is probably attributable to between-category confusions in the mixed-category condition. Because each letter was very similar to a digit, it may have been more difficult to identify items in the mixed-category condition than in the single-category condition.

Accuracies on present and absent trials are shown in Table 2. As with the $A^{\prime}$ scores, there was an effect of single versus mixed category $[F(1,9)=14.62, p<.005]$. There was no significant effect or interaction with the present versus absent factor. Response times are shown in Table 2 as well. The only significant effect was that absent responses took longer than present responses $[F(1,9)=34.92, p<.001]$.

\section{EXPERIMENT 3}

One possible objection to the conclusions of Experiment 2 is that the similarity between matched digits may not have equaled the similarity between matched digitletter pairs. For example, if the two digits in a group were much more confusable than each was with its corresponding letter, the single-category condition would be at a disadvantage relative to the mixed-category condition. In effect, the confusability would be greater within the digit category than between categories. In this case, the manipulation used in Experiment 2 would have overcompensated for the bias in favor of the single-category condition that was presumed to exist in Experiment 1.

Experiment 3 was designed to test for this possibility. Observers were shown two characters and were to respond same as quickly as possible if the characters were identical and different otherwise. The rationale was that different response time would be a function of visual similarity, with visually similar characters having longer response times than less similar characters (e.g., Cooper \& Podgorny, 1976). Thus, if it took longer to respond different to matched digit pairs (i.e., the upper row in each group in Figure 3) than to matched digit-letter pairs (i.e., the columns in each group), that would be evidence that the digit pairs were more similar than the digit-letter pairs. This would suggest that Experiment 2 was a biased test of the category effect. On the other hand, no difference between the different response times would suggest that the two pairs were equally similar and that the experiment was a fair test.

Other pairs of characters from within each group of four were included as additional different trials. These pairs were unmatched in the sense that there was no direct attempt to ensure that they differed by only a feature or two. For instance, the lower row in each group in Figure 3 comprises an unmatched letter pair. The similarity of these characters arises only because they are similar to similar digits; there was no attempt to manipulate their similarity directly. In addition, characters on the diagonal of each group comprise unmatched digit-letter pairs; these characters are similar only because they were selected to resemble a common digit. These unmatched pairs are likely to be less similar than the matched pairs, and consequently should have shorter different response times.

\section{Method}

On each trial, observers were shown two characters and asked to decide as quickly as possible whether they were the same or different. The procedure was as follows. When the computer was ready to begin a trial, it showed a fixation field consisting of a white rectangle $2.0^{\circ}$ wide and $1.4^{\circ}$ high. The observers began each trial by simultaneously pressing the two response switches in a hand-held response box. After $\mathbf{5 0 0} \mathrm{msec}$, two characters were shown centered in the fixation field $0.8^{\circ}$ apart. If the two characters were identical, the observers responded same by pressing the right-hand switch; otherwise, they responded different by pressing the left-hand button. The characters remained in view until a response was made, at which time the display was extinguished. The apparatus and viewing conditions were the same as in Experiments 1 and 2 .

Stimulus pairs for different trials were constructed by pairing each character with every other character in its group two times, once on the left and once on the right. This resulted in a total of 12 pairs for each group of 4 , or a total 48 pairs altogether. These 48 different pairs consisted of 8 matched digit pairs (the top row in each group of 4), 16 matched digit-letter pairs (the columns in each group), 8 unmatched letter pairs (the bottom row in each group), and 16 unmatched digit-letter pairs (the diagonals in each group). Thirty-two same pairs were constructed by twice pairing each of the 16 characters with itself. The observers, 10 undergraduates at the University of Alberta, participated in one practice block and eight test blocks in which these 80 stimulus pairs were presented in a random order.

\section{Results and Discussion}

Median correct response times are shown in Table 3 for each of the five types of stimulus pairs. As can be seen, there was virtually no difference between the time to respond different to matched digit pairs and matched digit-letter pairs $[t(9)=0.80]$. A $95 \%$ confidence inter-

Table 3

Response Times (in Milliseconds) and Percent Errors in Experiment 3

\begin{tabular}{lcc}
\hline \multicolumn{1}{c}{ Pair Type } & Response Time & Percent Errors \\
\hline Identical & 485 & 5.9 \\
Matched Digits & 527 & 6.1 \\
Matched Digit-Letter & 533 & 5.9 \\
Unmatched Letters & 502 & 1.7 \\
Unmatched Digit-Letter & 505 & 0.7 \\
\hline
\end{tabular}


val for this difference was quite small, extending from -11 to 24 msec. This result suggests that the digit pairs were no more visually similar than the matched digit-letter pairs, and that the matching manipulation was successful in controlling the visual similarity within and between categories. Thus, Experiment 2 was probably not biased against the single-category condition.

The power of this experiment to reveal effects of visual similarity is demonstrated by the results for the unmatched pairs. Although these pairs were not selected to differ minimally as the matched pairs were, they nevertheless were fairly similar. The response times shown in Table 3 indicate that in all cases the unmatched pairs had faster different response times than the matched pairs $[t(9)>$ $4.67, p<.005$, for all comparisons]. This indicates that the unmatched pairs are clearly less similar than the matched pairs. Thus, the same-different task is capable of detecting relatively subtle differences in similarity, and the failure to find a difference between matched digit and matched digit-letter pairs cannot be attributed to a lack of sensitivity.

The error rates are shown in Table 3. As with the response times, there was no significant difference between matched digits and the matched digit-letters, although both of the matched-pair types had significantly more errors than both of the unmatched-pair types $(p<.05$ in all cases, two-tailed sign tests).

\section{GENERAL DISCUSSION}

These experiments tested the adequacy of the partial analysis explanation of the category effect by matching letters and digits on visual similarity. In Experiment 1, a category effect was found with partial report (positive SOA) but not with visual detection (negative SOA). In Experiment 2, the structure of the stimulus set was modified to equalize similarities between and within categories, and a category effect was found in the visual detection task as well. Experiment 3 confirmed that the matched digit pairs and matched digit-letter pairs used in Experiment 2 were, in fact, equally similar, and that the stimuli were not biased in favor of finding a category effect. The overall conclusion seems to be that the category effect does not depend on physical feature differences between categories.

An alternative explanation of the category effect is that items are categorized in parallel without the use of processing resources. Attention can then be directed to the relevant item on the basis of category. Subsequent limited-capacity operations would be used to decide whether that item matched the target (as in a visual detection task) or to retain the item in memory (as in a partial-report task). At present it is not clear whether the early parallel processing of the items includes identification of the items as well as categorization. It might be argued that in the absence of simple feature differences between categories, an item must be at least implicitly identified before its category can be decided. On the other hand, Taylor (1978) found evidence suggesting that categorization and identification go on in parallel, with categorization sometimes preceding identification. Whatever the relationship between categorization and identification, the present results make it clear that categorization is not being done on the basis of simple feature differences.

The contrast between Experiments 1 and 2 emphasizes the importance of interitem similarity in the category effect. Whether the effect is obtained or not would seem to depend solely on the similarity of items within and between categories. This may account in part for the conflicting results sometimes obtained in category effect experiments. For instance, Francolini and Egeth (1979) failed to find a category effect in a task very similar to that used successfully by Taylor (1978), and Sperling (1960) failed to find a category effect in partial report, whereas Duncan (1983b) found a substantial one. Undoubtedly, methodological differences may account for some of these failures to replicate. However, it is also possible that the different character sets used in these experiments produced variations in visual similarity between and within categories, and that this variation contributed to whether or not a category effect was obtained.

In sum, two important conclusions can be drawn from the present experiments. First, the results demonstrate that interitem similarity is an important variable that needs to be considered in accounts of the category effect. Depending on how the stimuli are constructed, the results may be biased either for or against finding an effect. Second, the present results show that a category effect can occur when all of the simple feature differences between letters and digits have been controlled. Thus, in at least some situations, the effect depends on the conceptual distinction between the two categories rather than on any physical differences.

\section{REFERENCES}

Cooper, L. A., \& Podgorny, P. (1976). Mental transformations and visual comparison processes: Effects of complexity and similarity. Joumal of Experimental Psychology: Human Perception \& Performance, 2, 503-514.

Corcoran, D. W. S., \& JACKSON, A. (1977). Basic processes and strategies in visual search. In S. Dornic (Ed.), Attention and performance $V$ (pp. 387-411). Hillsdale, NJ: Erlbaum.

DeutsCh, J. A. (1977). On the category effect in visual search. Perception \& Psychophysics, 21, 590-592.

Di Lollo, V., \& Moscovitch, M. (1983). Perceptual interference between spatially separate sequential displays. Canadian Journal of Psychology, 37, 414-428.

Dixon, P. (1985). The category effect in visual detection and partial report. Perception \& Psychophysics, 38, 286-295.

Dixon, P. (1986). Attention and interference in the perception of brief visual displays. Joumal of Experimental Psychology: Human Perception \& Performance, 12, 133-148.

Duncan, J. (1980). The locus of interference in the perception of simultaneous stimuli. Psychological Review, 87, 272-300.

Duncan, J. (1983a). Category effects in visual search: A failure to replicate the "oh-zero" phenomenon. Perception \& Psychophysics, 34, 221-232.

DunCaN, J. (1983b). Perceptual selection based on alphanumeric class: 
Evidence from partial reports. Perception \& Psychophysics, 33, 533-547.

EGETH, H., JoNIDES, J., \& Wall, S. (1972). Parallel processing of multielement displays. Cognitive Psychology, 3, 674-698.

Francolini, C. M., EgeTh, H. E. (1979). Perceptual selectivity is task dependent: The pop-out effect poops out. Perception \& Psychophysics, 25, 99-110.

GARDNER, G. T. (1973). Evidence for independent parallel channels in tachistoscopic perception. Cognitive Psychology, 4, 130-155.

GREEN, D. M., \& SWETS, J. A. (1966). Signal detection theory and psychophysics. New York: Wiley.

GrIER, J. B. (1971). Nonparametric indexes for sensitivity and bias: Computing formulas. Psychological Bulletin, 75, 424-429.

INGLING, N. W. (1972). Categorization: A mechanism for rapid information processing. Journal of Experimental Psychology, 94, 239-243.

Jonides, J., \& GleItMAN, H. (1972). A conceptual category effect in visual search: $O$ as letter or as digit. Perception \& Psychophysics, $12,457-460$.

Kahneman, D., \& Treisman, A. (1984). Changing views of attention and automaticity. In R. Parasuraman, R. Davies, \& J. Beatty (Eds.), Varieties of attention (pp. 29-61). New York: Academic Press.

Krueger, L. E. (1984). The category effect in visual search depends on physical rather than conceptual differences. Perception \& Psychophysics, 35, 558-564.

MerIKLE, P. M. (1980). Selections from visual persistence by perceptual groups and category membership. Journal of Experimental Psychology: General, 109, 279-295.

Pollack, I., \& Norman, D. A. (1964). A nonparametric analysis of recognition experiments. Psychonomic Science, 1, 125-126.

SCHNEIDER, W., \& SHIFFrIN, R. M. (1977). Controlled and automatic human information processing: I. Detection, search and attention. Psychological Review, 84, 1-66.

SPERLNG, G. (1960). The information available in brief visual presentation. Psychological Monographs, 74(11, Whole No. 498).

TAYLOR, D. A. (1978). Identification and categorization of letters and digits. Joumal of Experimental Psychology: Human Perception \& Performance, 4, 423-439.

TOWNSEND, V. M. (1973). Loss of spatial and identity information following a tachistoscopic exposure. Journal of Experimental Psychol$o g y, 98,113-118$.

(Manuscript received July 24, 1986; revision accepted for publication May 18, 1987.)

\title{
Meeting Announcement
}

\author{
Visual Form and Motion Perception: Psychophysics, Computation, and Neural Networks \\ Friday and Saturday, March 4 and 5, 1988 \\ Conference Auditorium, George Sherman Union, Boston University \\ 775 Commonwealth Avenue, Boston, Massachusetts
}

This meeting has been dedicated to the memory of the late KVETOSLAV PRAZDNY, who was to have been a speaker, and whose tragic death has deprived the field of visual perception of one of its most talented investigators.

Speakers include: L. Arend, Eye Research Institute; S. Anstis, York University; I Biederman, University of Minnesota; P. Cavanagh, University of Montreal; J. Daugman, Harvard University; S. Grossberg, Boston University, J. Lappin, Vanderbilt University; E. Mongolla, Boston University; V. Ramachandran, UCSD; A. Reeves, Northeastern University; W. Richards, MIT; R. Savoy, Rowland Institute; G. Sperling, New York University; J. Todd, Brandeis University; S. Zucker, McGill University.

This meeting is sponsored by the Boston Consortium for Behavioral and Neural Studies, a group of researchers supported by the Air Force Office of Scientific Research Life Sciences Program.

A Howard Johnson's Motor Lodge is located at 575 Commonwealth Avenue, and a limited number of rooms at a reduced conference rate can be reserved until February 10, 1988, by those attending the meeting. Total conference registration will be limited by available meeting space, so early registration is advised.

Registration and hotel accommodations for the meeting are being handled by UNIGLOBE-Vision Meeting, 40 Washington Street, Wellesley Hills, MA 02181 (Telephones: (800)521-5144, (617)2357500). To register for the meeting or for further information about travel or accommodation arrangements, contact UNIGLOBE. 